\title{
Blue nevus with satellitosis: case report and literature review*
}

\author{
Ana Helena Kalies Oliveira ${ }^{1}$ \\ Ana Flávia de Melo Cavalcanti Shiraishi ${ }^{3}$ \\ Bogdana Victoria Kadunc ${ }^{2}$
}

\author{
Patrícia de Carvalho Sotero ${ }^{2}$ \\ Rafael Fantelli Stelini ${ }^{4}$ \\ Cínthia Mendes ${ }^{5}$
}

DOI: http://dx.doi.org/10.1590/abd1806-4841.20175267

\begin{abstract}
Blue nevus is a benign melanocytic lesion, typically asymptomatic and of unknown etiology. Many histological subtypes are recognized, the most commons being: common blue nevus, cellular blue nevus, and combined blue nevus. New rare variants have been described in the literature, with emphasis on eruptive blue nevus, plaque, agminate, linear, with satellitosis, disseminated, familial and targetoid. The diagnosis of blue nevus usually presents no difficulties, however, the presence of structures such as irregular edges or satellitosis, are highly suggestive of malignancy, and the differential diagnosis with malignant blue nevus and melanoma with peripheral spread should be considered. We report a case of blue nevus with satellitosis in a 15-year-old female patient.
\end{abstract}

Keywords: Dermoscopy; Diagnosis, differential; Melanoma; Nevus, blue; Satellite

\section{INTRODUCTION}

Blue nevus is a benign melanocytic lesion of unknown etiology. It is believed to occur by the deposition of melanocytes in the dermis during a defective embryonic migration process of these cells from the neural crest to the epidermis. The characteristic blue color is due to the pigmentation of melanocytes localized in the mid-dermis, where longer wave lengths are absorbed and the shorter wave lengths are reflected, corresponding to the color blue - known as Tyndall effect. ${ }^{1}$

Blue nevus is more common in adults in the second decade of life, and can present as a pigmented papule, macule, plaque or nodule. It is more frequently found as a dark blue or black-blue solitary lesion. ${ }^{1,2}$ On dermoscopy, it appears as a pigmented lesion, in a homogenous pattern, with structureless or amorphous areas (more common), or with dots and globules. It preferentially affects the dorsum of hands and feet, face, scalp, buttocks and, rarely, mucosa. Some histological variants are described, the most commonly found being common blue nevus, cellular blue nevus and combined blue nevus. Other rarer subtypes have been described, such as plaque blue nevus, with satellitosis, linear, eruptive and agminate. ${ }^{1}$
The diagnosis of blue nevus is usually not difficult, however, the presence of structures like irregular borders or satellitosis is highly suggestive of malignant lesions and the differential diagnosis with malignant blue nevus and melanoma must be considered. ${ }^{3-5}$ Few cases of benign blue nevus with satellite lesions were reported; this is the eighth case reported. ${ }^{2-8}$

\section{CASE REPORT}

A 15-year-old female patient presented to us with a pigmented lesion on the lateral aspect of the left arm for 1 year, slowly enlarging and itchy over the past 2 months (Figure 1). Dermatological examination revealed a brown-blue irregular, well defined macule, with approximately $8 \mathrm{~mm}$, with black-brown dots on the periphery (Figure 2). On dermoscopy, there was a black-brown lesion, in a homogenous pattern, composed of structureless areas and with streaks in the periphery and satellite gray-blue lesions (Figure 3). Due to the suspicion of melanoma, an excisional biopsy was performed. Histopathology revealed spindle-shaped and dendritic, strongly pigmented melanocytes, amid dense fibrous stroma

Work submitted on 20.10.2015

Approved by the Advisory Board and accepted for publication on 22.10.2016

* Study conducted at Hospital da Pontifícia Universidade Católica de Campinas (Hospital e Maternidade Celso Pierro). Financial support: None

Conflict of interest: None

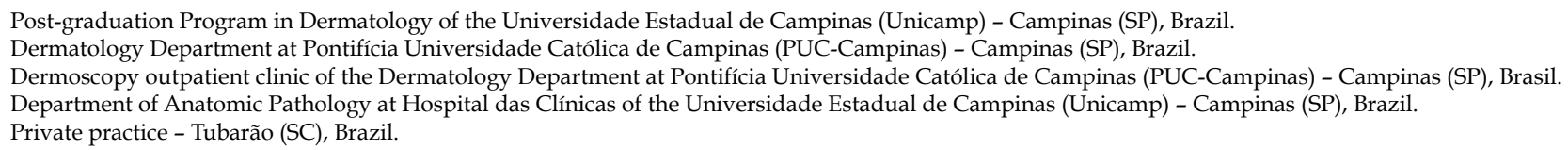


and melanophages, predominantly occupying upper and mid dermis (Figure 4). Satellite lesions were seen in the adjacent skin, constituted by melanocytes similar to the central lesion, forming small aggregates located close to the dermal vessels (Figure 5). The diagnosis was of common blue nevus with satellitosis.

\section{DISCUSSION}

One of the rare variants of blue nevus is blue nevus with satellitosis. The term "satellite" is defined as a skin cancer that disseminated from the primary tumor through the lymphatic system to up to $2 \mathrm{~cm}$ from the original tumor. For benign tumors such as blue nevus, the term "satellite" is used regardless the dissemination route of the lesion cells or the distance between the satellite and the main tumor; these cases refer to the clinically visible lesion, located on the skin close to the main lesion. ${ }^{9}$ Satellite lesions occur more commonly in malignant neoplasias, therefore the differential diagnosis of blue nevus with satellitosis is melanoma with peripheral spread and malignant blue nevus - rare melanoma variant that evolves from a preexisting common cellular blue nevus or that mimics a cellular blue nevus with no clear association with the precursor lesion. ${ }^{1}$ These hypotheses must be ruled out with the help of history and physical examination, associated to dermoscopy and, specially, the excisional biopsy, which is mandatory in lesions that are growing, that present with ulceration and a diameter larger than $1 \mathrm{~cm}$, that have an atypical dermoscopic appearance or satellitosis. ${ }^{3-5,7}$

Kang \& Chung (1999), in a case report of common blue nevus with satellite lesions, demonstrated that the satellite lesions

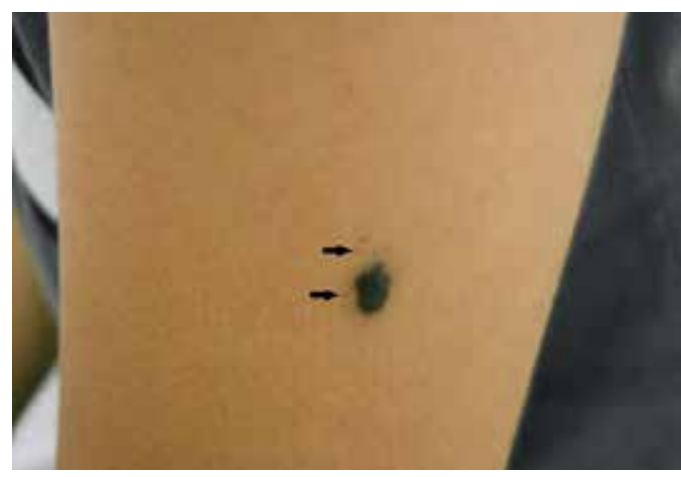

Figure 1: Pigmented lesion on the lateral aspect of the left arm

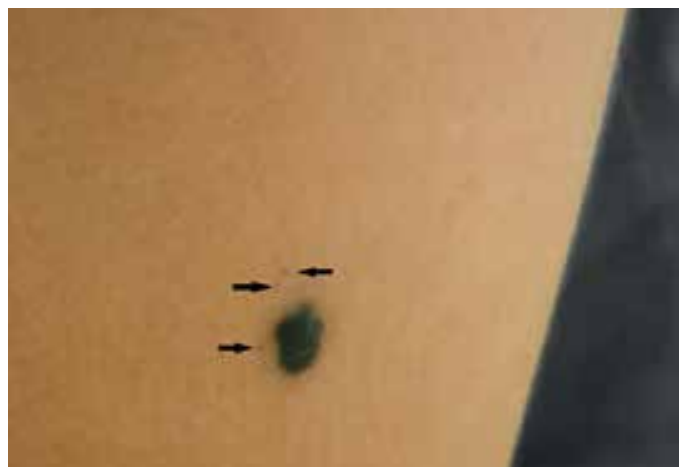

Figure 2: Close up of pigmented lesion on the left arm with satellite lesions were identical to the main blue nevus and were grouped around blood vessels, linearly arranged along the course of the vessels, as we observed in the satellite lesions in our case. They also showed nevus cells infiltrating blood vessel walls in both the main and satellite lesions, suggesting a possible spread of the benign nevus cells via the perivascular space. This type of spread would result in a very rare clinical manifestation for common blue nevus, erroneously suggestive of melanoma. ${ }^{2}$

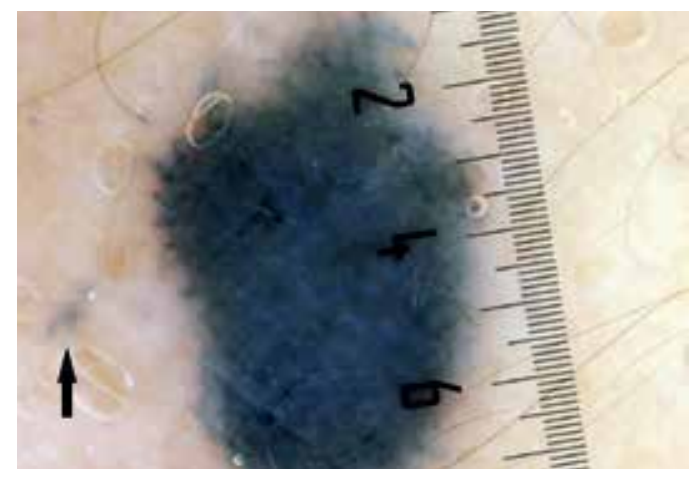

Figure 3: Dermoscopy: blue-gray in a homogenous pattern, with peripheral streaks and satellite lesions



Figure 4: Central lesion showing strongly pigmented melanocytes amid dense fibrous stroma and melanophages (Hematoxylin \& eosin, X40)

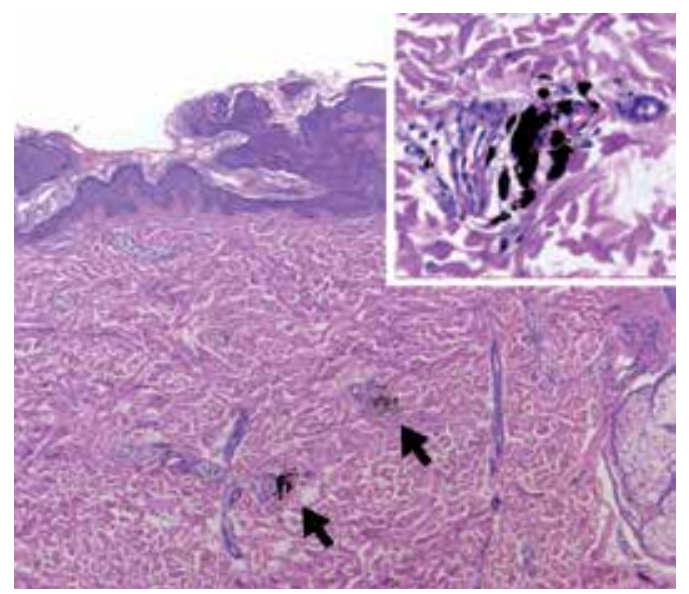

Figure 5: Satellite lesions composed by melanocytes similar to the central lesion, located close to the vessels in the dermis (black arrows) (Hematoxylin \& eosin, panoramic: X100; in set: X400) 
TABLE 1: Main findings of the case reports found in the literature

\section{Year of Age Sex Location Size}

report

1

199931 Female Forearm $8 \mathrm{~mm}$

22000

66

Male

Scalp

$18 \mathrm{~mm}$ Malignant mela-

$x$ noma; common

$15 \mathrm{~mm}$ blue nevus

32001

$70 \quad$ Male Scalp

$8 \mathrm{~mm}$ Melanoma; malignant blue nevus
$72013 \quad 24$ Male Forearm $10 \mathrm{~mm}$ Malignant mela$\mathrm{x}$ noma; common

$7 \mathrm{~mm}$ blue nevus

$8 \quad 2015 \quad 15 \quad$ Female Arm $8 \mathrm{~mm}$ Melanoma

\section{Dermoscopic \\ examination}

Not described Nevus cells in the papillary and reticular layers of the dermis, more concentrated in the perivascular and perilymphatic regions

Not described Elongated melanocytes in the reticular dermis

Homogenous Spindle-shaped cells with abundant melanophages color and regular shape

Not described Nodular dermal proliferation of
interspersed with dendritic cells

Not described Strongly pigmented oval-shaped, spindle-shaped and dendritic melanocytes and melanophages

Homogenous Spindle-shaped cells with abundant melanophages color nodule and regular shape

Blue-white structure, homogenous

Hyperpigmented spindle-shaped melanocytes, spread in the dermis and among the collagen, more concentrated in the perivascular and perilymphatic regions

Homogenous pattern with structureless areas and peripheral streaks
Spindle-shaped and dendritic hyperpigmented melanocytes, amid dense fibrous stroma and melanophages, occupying upper and mid dermis. Satellite lesions composed of melanocytes similar to the main lesion, located close to the vessels in the dermis
The presence of blue nevus melanocytes in the wall of peripheral nerve fibers is also described. We speculate that this could be another possibility of nevus cells spread in cases of blue nevus with satellitosis. Besides, this association with nerve fibers and the demonstration of structures similar to nerve fibers - neuroid figures - inside some blue nevi led some authors to suggest that the cellular formation of these nevi takes place inside a nerve sheath. ${ }^{10}$

According to a search in Lilacs, Medline and Pubmed databases, there are 7 cases of blue nevus with satellitosis reported up to now, ${ }^{2-8}$ making this the eighth report. Table 1 shows the main findings in these cases. There is a wide variation in patients (from 15 to 71 years old); ours is the youngest patient described. There seems to be a predilection for the scalp and upper limbs, and the lesions are, in average, $9.5 \mathrm{~mm}$ in size (ranging from $6 \mathrm{~mm}$ to $18 \mathrm{~mm}$ ).
In almost all reports, the main hypothesis was of a malignant lesion, such as melanoma or malignant blue nevus. These were ruled out only after histology, that did not show any features of malignant behavior such as mitoses, atypia or nuclear pleomorphism. Before the histopathology, blue nevus was considered in only two cases.

Such as in the other cases, this had clinical and dermoscopic elements that suggested malignancy, such as radial streaks and satellite lesions. With the microscopic analysis, the diagnosis of common blue nevus with satellitosis was confirmed. Therefore, we highlight the importance of the differential diagnosis with melanoma, based in the correlation of clinical, dermoscopic and microscopic data. $\square$ 


\section{REFERENCES}

1. Araújo CSA, Melhado RM, Sereno VJ, Belice APN, Lopes LC, Gonçalves KJ. Nevo Azul: relato de caso clínico. Rev. Omnia Saúde. 2007;4:42-5.

2. Kang DS, Chung KY. Common blue naevus with satellite lesions: possible perivascular dissemination resulting in a clinical resemblance to malignant melanoma. Br J Dermatol. 1999;141:922-5.

3. Sahin MT, Demir MA, Yoleri L, Can M, Oztürkcan S. Blue naevus with satellitosis mimicking malignant melanoma. J Eur Acad Dermatol Venereol. 2001;15:570-3.

4. Yonei N, Kimura A, Furukawa F. Common Blue Nevus with Satellite Lesions Needs a Differential Diagnosis from Malignant Melanoma. Case Rep Dermatol. 2013;5:244-7.

5. del Río E, Vázquez Veiga HA, Suárez Peñaranda JM. Blue nevus with satellitosis mimicking malignant melanoma. Cutis. 2000;65:301-2.

6. Lourari S, Lamant L, Viraben R, Paul C, Meyer N. Photoletter to the editor: blue nevus with satellitosis mimicking melanoma. Contribution of dermoscopy and reflectance confocal microscopy. J Dermatol Case Rep. 2012;6:54-6.

7. Piana S, Grenzi L, Albertini G. Cellular blue nevus with satellitosis: a possible diagnostic pitfall. Am J Dermatopathol. 2009;31:401-2.

8. Knopp E, Diette K, Ko C, Lazova R. Multiple blue macules and papules on the scalp. Arch Dermatol. 2009;145:1183-8.

9. Edge SB, Compton CC. The American Joint Committee on Cancer: the 7th Edition of the AJCC Cancer Staging Manual and the Future of TNM. Ann Surg Oncol. 2010;17:1471-4.

10. Misago N. The relationship between melanocytes and peripheral nerve sheath cells (Part II): blue nevus with peripheral nerve sheath differentiation. Am J Dermatopathol. 2000;22:230-6.
MAILING ADDRESS:

Ana Helena Kalies Oliveira

Av. John Boyd Dunlop, $\mathrm{S} / \mathrm{N}$

Jardim Londres

13034-685 Campinas, SP

Brazil

E-mail:ahkalies@gmail.com

How to cite this article: Oliveira AHK, Sotero PC, Shiraishi AFMC, Stelini RF, Kadunc BV, Mendes C. Blue nevus with satellitosis: case report and literature review. An Bras Dermatol. 2017;92(5 Suppl 1): 30-3. 